\title{
Seleção de espécies bioindicadoras da presença de tebuthiuron no solo
}

\section{Selection of indicative species of the presence of tebuthiuron in the soil}

\author{
João Henrique dos Santos Ferreira, \\ Universidade Federal do Triângulo Mineiro \\ E-mail: joaocellof1998@gmail.com \\ OrcID: https://orcid.org/0000-0002-0067-5628 \\ Matheus Ceolin Marques Queiroz \\ Universidade Federal do Triângulo Mineiro \\ E-mail: matheusceolinmq@gmail.com \\ OrcID: https://orcid.org/0000-0002-7669-0170 \\ Ilca Puertas Freitas e Silva \\ Universidade Estadual de Mato Grosso do Sul \\ E-mail: ilcapuertas@gmail.com \\ OrcID: https://orcid.org/0000-0001-8212-9905 \\ Christiane Augusta Diniz Melo \\ Universidade Federal do Triângulo Mineiro \\ E-mail: christiane.melo@uftm.edu.br \\ OrcID: https://orcid.org/0000-0001-6385-2153
}

Resumo: O herbicida tebuthiuron é utilizado em cultivos de cana-de-açúcar sendo um produto persistente no solo. Assim, objetivou-se avaliar a suscetibilidade de diferentes espécies a doses do tebuthiuron para a seleção de plantas bioindicadoras. O experimento foi conduzido em casa de vegetação, em delineamento inteiramente casualizado com quatro repetições, utilizando-se um Latossolo Vermelho-Amarelo distrófico de textura franco-arenosa. Os tratamentos constaram de doses do tebuthiuron: 0,$0 ; 0,12 ; 0,24 ; 0,48 ; 0,72 ; 0,96$; $1,2 \mathrm{~kg} \mathrm{ha}^{-1}$ i.a.. As espécies testadas foram alface, amendoim, aveia, beterraba, braquiária, crotalária, pepino, sorgo e tomate. Aos 7, 14 e 21 dias após emergência (DAE) foram realizadas avaliações de fitotoxicidade e aos 21 DAE fez-se a determinação da massa da matéria seca da parte aérea. Por demonstrarem sensibilidade diferencial em relação às doses, em ordem crescente de sensibilidade: amendoim, aveia, braquiária, sorgo e pepino apresentam potencial para uso como bioindicadoras em estudos com tebuthiuron no solo.

Palavras-chave: Bioensaio. Comportamento de herbicida no solo. Herbicida.

Abstract: The herbicide tebuthiuron is used in sugarcane crops and it is a persistent in the soil. Thus, objective was to evaluate the susceptibility of different species for the selection of bioindicatorplants to doses of tebuthiuron. The experiment was carried under greenhouse conditions, in a completely randomized design with four replications, using a sandy-loam texture Oxisol. Doses of tebuthiuron $(0,0 ; 0,12 ; 0,24 ; 0,48 ; 0,72$; 0,$96 ; 1,2 \mathrm{~kg} \mathrm{ha}^{-1}$ a.i.) were treatments. Beet, bread grass, cucumber, lettuce, oat, peanut, sorghum, sunn hemp and tomato were the tested species. Phytotoxicity assessments were evaluated at 7, 14 and 21 days after emergence (DAE) andat $21 \mathrm{DAE}$, the dry matter mass of the aerial partwas determined. As the demonstration of differential sensitivity in relation to doses, in an increasing order of sensitivity: peanut, oat, bread grass, sorghum and peanut, have potential in studies with bioindicators of tebuthiuron in soil.

Keywords: Bioassay. Herbicide. Herbicide behavior in the soil. 
O controle químico com a utilização de herbicidas na cultura da cana-de-açúcar é justificado pela eficácia no controle de plantas daninhas, baixa necessidade de mão- de- obra, rapidez e praticidade na aplicação (Marchesan, Dedordi, Rezzi, Vidal e Dick, 2011). Ademais, as características da cultura, que possui um crescimento inicial muito lento, fazem com que o período crítico de prevenção da interferência (PCPI) da cana-de-açúcar seja elevado, cerca de até 130 dias após o plantio, para cana planta de ano e meio (Hijano, 2016), justificando também a utilização de herbicidas com longo efeito residual, ou seja, herbicidas que controlam as plantas daninhas por um determinado período de tempo (Perim et al., 2009), como o tebuthiuron.

O herbicida tebuthiuron (N-[5-(1,1-dimetiletil)-1,3,4-tiadiazol-2-il]-N,N'-dimetilureia) é um dos produtos pertencentes ao mecanismo de ação dos inibidores do fotossistema II aplicados na cana-de-açúcar (Gomes, Spadotto, Pereira, Matallo e Luchini, 2006). É recomendado para aplicação em pré-emergência de plantas daninhas, mono e eudicotiledôneas, no cultivo de cana-de-açúcar (Pires et al., 2003). Este ingrediente ativo possui caráter não iônico, Kow $=61,7\left(\mathrm{pH}=7,0\right.$ e $\left.20^{\circ} \mathrm{C}\right)$ e alta solubilidade em água $=2500 \mathrm{mg} \mathrm{L}^{-1}\left(20^{\circ} \mathrm{C}\right)$ (The Pesticide Properties Data Base [PPDB], 2019).

Haja vista os benefícios da utilização do herbicida em lavouras, este tem grande potencial de impacto ambiental, quando o manejo é inadequado (Barizon, Lavorenti, Reginato, Prata e Tornisielo, 2006). A permanência do tebuthiuron no ambiente por um período maior que o PCPI é indesejável, por causar problemas como intoxicação de organismos não alvo (Faria et al., 2014), e possibilidade de contaminação de águas subterrâneas (Gomes, Spadotto e Lanchotte, 2001), carryover (Cadersa e Gungadurdoss, 2010) e dificuldade de cultivo de espécies sensíveis em sucessão (Fernandes, et al 2011).

Em trabalho realizado avaliando a persistência do tebuthiuron utilizando a aveia como planta bioindicadora, constatou-se que este pode persistir no ambiente por um período de aproximadamente 11 a 14 meses em Latassolo vermelho-amarelo (Blanco e Oliveira, 1987). Para Faria (2013) a meia-vida do tebuthiuron em Latossolo com pH 5,0 foi de 155 dias após aplicação e de 117 dias no mesmo solo, em pH 5,9. Faria et al. (2018), constataram-se alta capacidade de lixiviação do tebuthiuron, sendo detectada presença do herbicida em amostras coletadas em até $50 \mathrm{~cm}$ de profundidade em Latossolos.

Com o objetivo de estudar o comportamento de herbicidas no solo a técnica do bioensaio tem sido amplamente utilizada (Freitas et al., 2014; Guerra et al., 2011; Mendes et al., 2012), assim como análises laboratoriais de espectrometria de massa e cromatografia (Vidal \& Vidal, 2009). Apesar de eficientes, as análises laboratoriais são onerosas, e demandam mão de obra especializada (Walorczyk et al., 2013), e apresentam um limite de quantificação abaixo do qual não é possível quantificar o herbicida presente na amostra. Com o bioensaio é possível a detecção de resíduos biologicamente ativos dos produtos no solo, bem como observar alteração no crescimento inicial das bioindicadoras mesmo na presença de baixas concentrações de herbicida no solo (Hutchinson et al., 2007).

Para o emprego da técnica de bioensaio faz-se necessária a seleção de espécies vegetais que exibam sintomas visuais de toxicidade pela absorção do produto e, ou alteração do acúmulo de matéria seca e do crescimento inicial (Nunes e Vidal, 2009; Marchesan et al., 2011; Diesel et al., 2012).

Uma espécie bioindicadora da presença de herbicidas muito utilizada em diversos trabalhos é Cucumis sativus L. (pepino), a qual demonstra-se altamente sensível a diversos mecanismos de ação e grupo químicos de herbicidas, a exemplo dos herbicidas imazethapyr (Gazziero, Karan, Voll e Ulbrich, 1997), trifloxysulfuron-sodium e pyrithiobbac-sodium (Guerra et al., 2011), metribuzim (Mendes et al., 2015) e tebuthiuron (Guerra et al., 2016).

Outras espécies também foram utilizadas em experimentos de comportamento de herbicidas no solo, pela técnica de bioensaio, tais como: beterraba para estudos de persistência do sulfentrazone (Blanco e Velini, 2005); milheto como bioendicadora da presença de sulfentrazone (Madalão, Pires, Chagas, Cargnelutti Filho e Procópio, 2012), sorgo como bioendicadora dos herbicidas sulfentrazone, isoxaflutole e oxyfluorfen (Melo et al., 2010) e capim-braquiária para os herbicidas clomazone e diuron (Mendes et al., 2012).

Considerando a persistência do herbicida tebuthiuron no ambiente, associado a importância de estudos sobre o comportamento deste produto no solo, objetivou-se com este trabalho avaliar a suscetibilidade de diferentes espécies a doses do herbicida tebuthiuron para a seleção de plantas com potencial de uso como bioindicadoras.

\section{Material e Métodos}


O experimento foi conduzido em casa de vegetação no município de Iturama - MG, a $453 \mathrm{~m}$ altitude, $19^{\circ} 43^{\prime} 41.2^{\prime \prime S}$ e $50^{\circ} 13^{\prime} 59.9^{\prime \prime} \mathrm{W}$, sob delineamento inteiramente casualizado com quatro repetições.

Os tratamentos foram constituídos por sete doses do herbicida tebuthiuron 0,$0 ; 0,12 ; 0,24 ; 0,48 ; 0,72$; 0,96; $1,2 \mathrm{~kg} \mathrm{ha}^{-1}$ i.a., correspondentes a, respectivamente, $0,10,20,40,60,80$ e $100 \%$ da dose recomendada. As espécies testadas foram Lycopersicon esculentum Mill. (tomate), Cucumis sativus L. (pepino), Beta vulgaris L. (beterraba), Lactuca sativa L. (alface), Sorghum bicolor (L.) Moench. (sorgo), Arachis hypogaea L. (amendoim), Avena sativa L. (aveia), Urochloa brizantha (Hochst.) Stapf. (braquária) e Crotalaria juncea L. (crotalária).

O LATOSSOLO VERMELHO-AMARELO Distrófico (Santos et al., 2018) de textura franco-arenosa, oriundo da região do Pontal do Triângulo, Mineiro foi utilizado para preencher os vasos de $500 \mathrm{~cm}^{3}$, após caracterização físico-química (Tabela 1). Após enchimento dos vasos, procedeu-se ao umedecimento do solo próximo a capacidade de campo, realizou-se a semeadura das espécies bioindicadoras e logo em seguida o herbicida tebuthiuron foi aplicado nas doses estabelecidas, utilizando pulverizador costal pressurizado com $\mathrm{CO}_{2}$ a $200 \mathrm{kPa}$, barra com ponta de jato plano (tipo "leque") 110.02 a 0,5 m da superfície do solo e volume de calda de $200 \mathrm{~L} \mathrm{ha}^{-1}$.

Tabela 1. Caracterização química do solo.

\begin{tabular}{|c|c|c|c|c|c|c|}
\hline$\overline{\mathrm{pH}}$ & $\bar{P}$ & $\mathrm{H}^{+}+\mathrm{Al}^{3+}$ & $\mathrm{Al}^{3+}$ & $\mathrm{Ca}^{2+}+\mathrm{Mg}^{2+}$ & $\mathrm{Ca}^{2+}$ & $\mathrm{K}^{+}$ \\
\hline $\mathrm{CaCl}_{2}$ & $\mathrm{~g} \mathrm{dm}^{-3}$ & & ---------- & $\mathrm{nol}_{\mathrm{c}} \mathrm{dm}^{3}-$ & & \\
\hline 4,5 & 2 & 0 & 32 & 31 & 41 & 4 \\
\hline${ }^{(1)} \mathrm{SB}$ & ${ }^{(2)} \mathrm{CTC}$ & ${ }^{(3)} \mathrm{V}$ & M.O. & Areia & Silte & Argila \\
\hline ------mr & | & $\%$ & $\mathrm{~g} \mathrm{dm}^{-3}$ & & $\%$ & \\
\hline 45 & 75 & 26 & 22 & 69,1 & 7 & 23,9 \\
\hline
\end{tabular}

Resina, M.O.: Ac. Sulfúrico, H+Al: Tampão SMP, Al: Fosf. Cálcio (Teixeira, Donagemma, Fontana e Teixeira, 2017).

Após o desbaste foram deixadas três plantas para as espécies eudicotiledôneas e quatro para as monocotiledôneas por vaso. As irrigações foram realizadas diariamente mantendo o solo próximo à capacidade de campo.

Aos 7, 14 e 21 dias após a emergência (DAE), realizaram-se avaliações visuais de fitotoxicidade, com uso de uma escala percentual de notas, variando entre 0 e $100 \%$, na qual as notas são referentes à porcentagem de área foliar afetada por injúrias ocasionadas pelo herbicida em relação à área foliar total da planta, em que 0\% representa a ausência de injúrias e 100\% a morte da planta (Sociedade Brasileira da Ciência das Plantas Daninhas [SBCPD], 1995).

Aos 21 DAE realizou-se o desmonte do experimento, sendo a parte aérea cortada rente ao solo para determinação da massa da matéria seca da parte aérea (MSPA), após secagem em estufa a $65{ }^{\circ} \mathrm{C}$ até peso constante.

Os valores da MSPA foram convertidos em porcentagem em relação à testemunha. Todos os dados foram submetidos à análise de variância, utilizando-se o teste $F(p<0,05)$. Posteriormente, ajustaram-se as equações de regressão por meio do pacote estatístico Sigmaplot 12.0 (2011). Os modelos de regressão foram escolhidos considerando-se a significância dos coeficientes de regressão e a explicação do fenômeno biológico. De posse das curvas, determinou-se a $\mathrm{GR}_{50}$, dose do herbicida tebuthiuron necessária para reduzir $50 \%$ do acúmulo da MSPA das plantas.

\section{Resultados e Discussão}

Observou-se níveis diferenciais de sensibilidade ao tebuthiuron (Figura 1), visualizados por meio do ajuste dos dados de fitotoxicidade nas curvas sigmoidais. O aumento da fitotoxicidade foi proporcional ao incremento das doses do herbicida tebuthiuron, havendo variação significativa desta variável em todas as espécies testadas, exceto para alface e beterraba que se mostraram extremamente sensíveis ao herbicida, morrendo todos os espécimes desde a dose mais baixa $\left(0,12 \mathrm{~kg} \mathrm{ha}^{-1}\right)$ em todas as avaliações realizadas após a emergência. 


\section{(a)garianin}

Alface (Lactuca sativa)

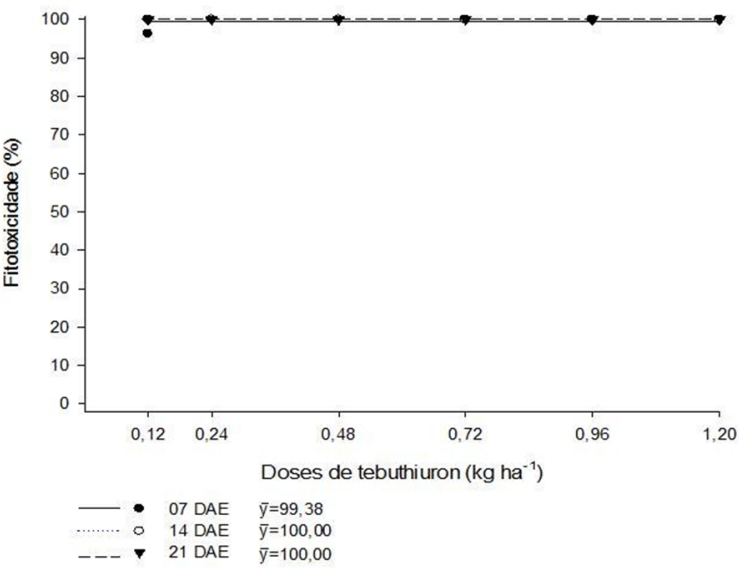

Aveia (Avena sativa)



Braquiária (Urochloa brizantha)

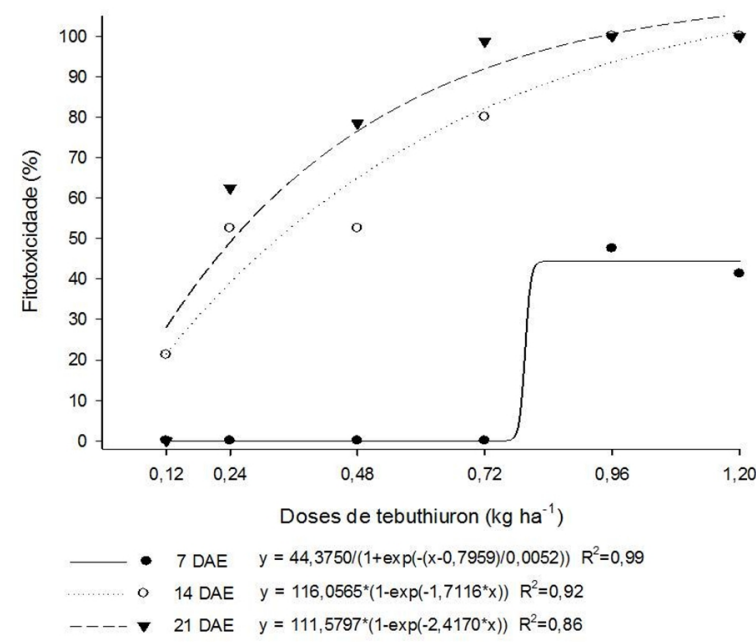

Pepino (Cucumis sativus)
Amendoim (Arachis hypogaea)

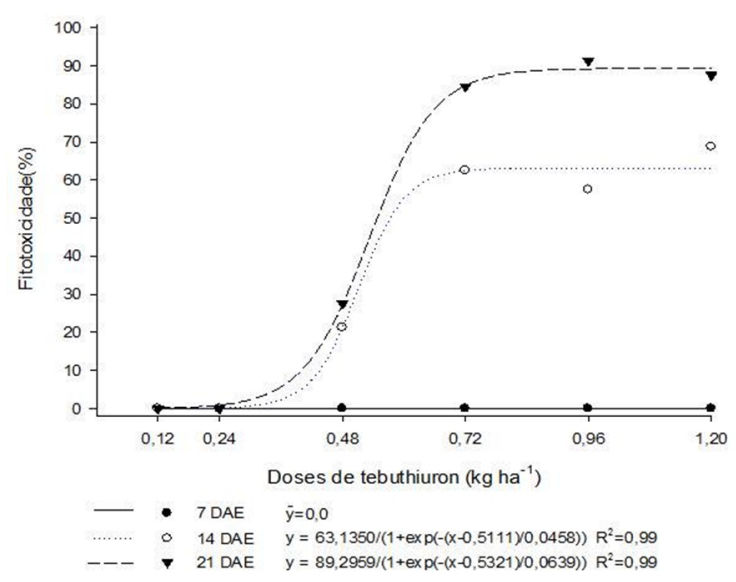

Beterraba (Beta vulgaris)

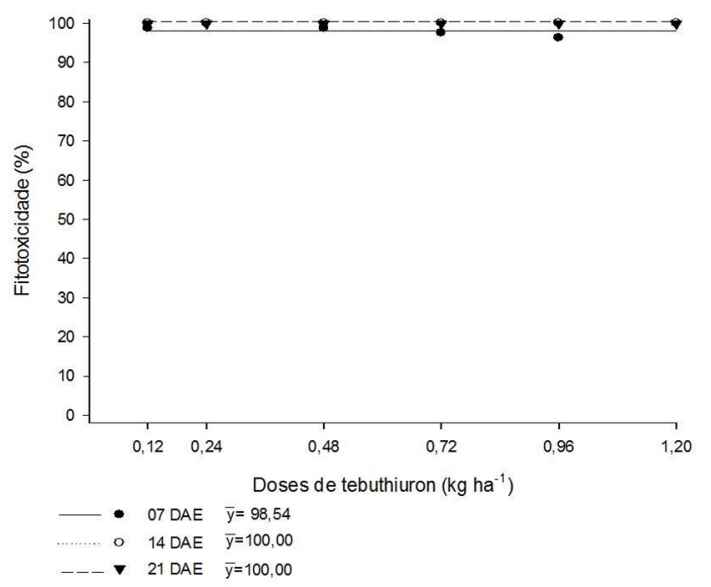

Crotalária (Crotalaria juncea)

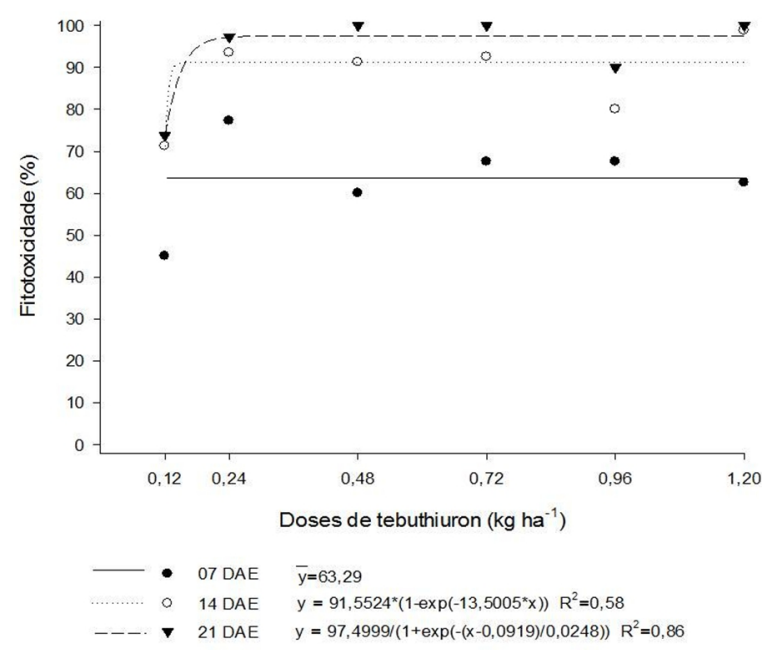

Sorgo (Sorghum bicolor) 


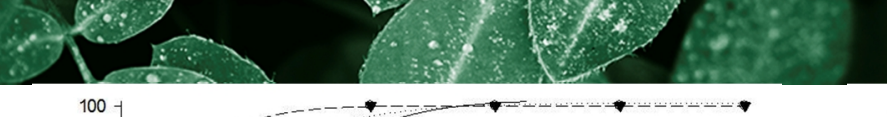
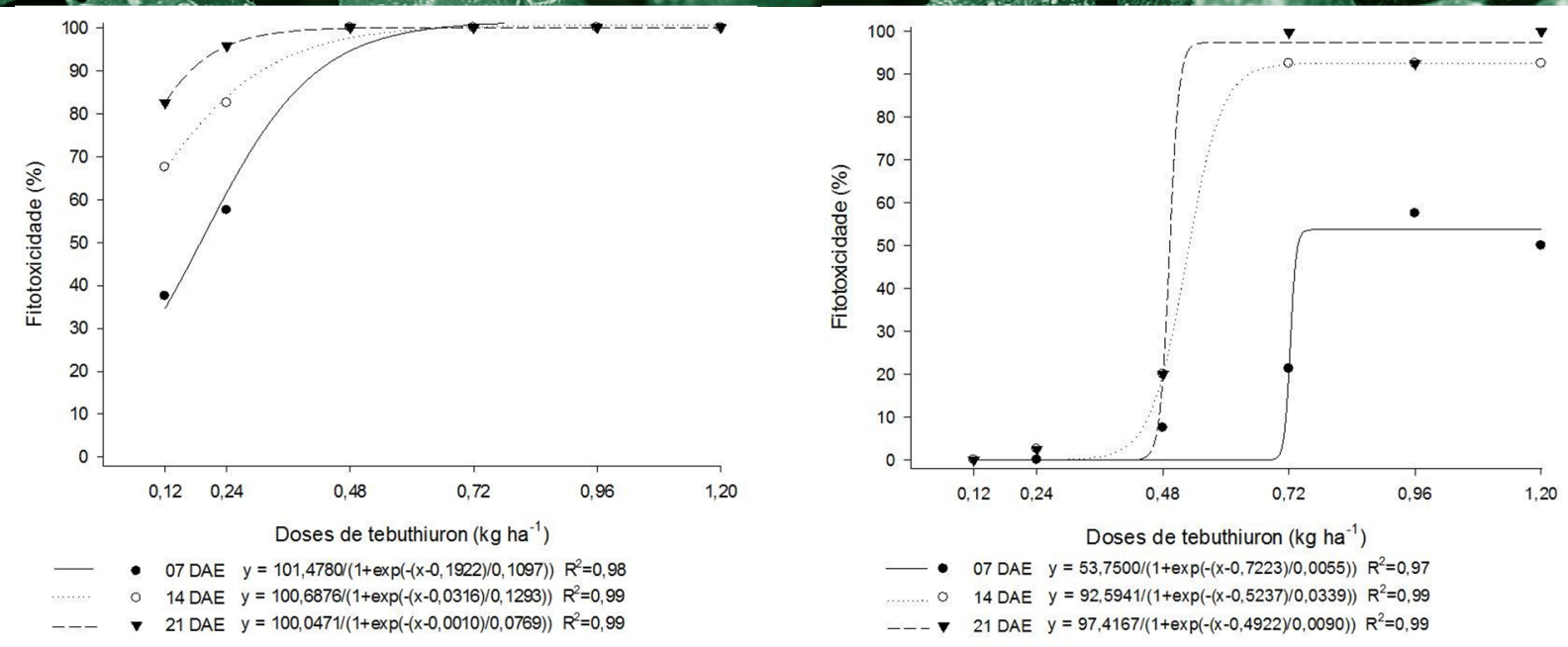

Tomate (Lycopersicon esculentum)

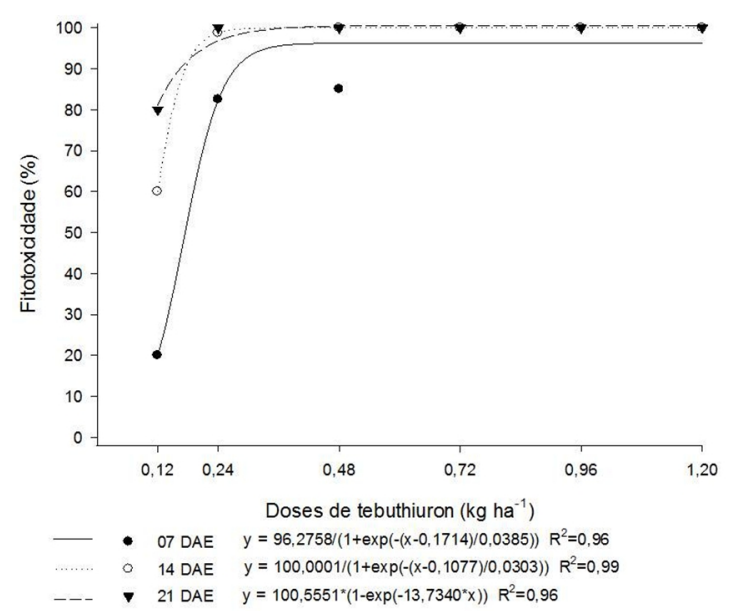

Figura 1. Fitotoxicidade observada em plantas de alface, amendoim, aveia, beterraba, braquiária, crotalária, pepino, sorgo e tomate $10 ; 20 ; 40 ; 60 ; 80$ e $100 \%$ da dose recomendada de tebuthiuron $\left(1,2 \mathrm{~kg} \mathrm{ha}^{-1}\right)$.

O tomate e aveia exibiram intoxicação severa aos 14 e 21 DAE respectivamente, verificando morte total das plantas a partir da dose de $0,24 \mathrm{~kg} \mathrm{ha}^{-1}$ (Figura 1). Em trabalho realizado por Peñaherrera-colina, Souza, Guilherme e Bueno Filho (2005), constatou-se que a aveia também se mostrou altamente sensível ao efeito do herbicida diuron, pertencente ao mesmo mecanismo de ação do tebuthiuron.

Quanto ao pepino, para a dose $0,48 \mathrm{~kg} \mathrm{ha}^{-1}$, aos 14 DAE e $21 \mathrm{DAE}$, constatou-se $98 \%$ e $100 \%$ de fitotoxicidade, respectivamente (Figura 1). Diversos trabalhos realizados a fim de estudar a dinâmica do herbicida tebuthiuron utilizaram-se o pepino (C. sativus) como planta bioindicadora (Silva Junior, Gonçalves, Queiroz e Martins, 2018; Guerra et al., 2016), diante da elevada sensibilidade da espécie. Contudo, tal sensibilidade mesmo em baixas doses pode dificultar a utilização da espécie em determinados estudos com a utilização de doses mais elevadas ou na quantificação indireta do produto presente no solo, por meio de curvas de dose-resposta, avaliando-se a variável massa da matéria seca, já que não apresenta uma tolerância diferencial, o que inviabilizaria a obtenção de dados, devido a elevada intoxicação da espécie mesmo em doses muito inferiores a recomendada do produto.

Conforme Nunes \& Vidal (2009), a espécie C. sativus demonstrou elevada sensibilidade a atrazine e metribuzin, herbicidas inibidores do FSII, impossibilitando a detecção das variáveis analisadas no trabalho, como altura e área foliar. Em Inoue et al. (2011), o herbicida ametryn propiciou controle superior $80 \%$ de $C$. sativus mesmo aos 40 dias após tratamento com herbicida.

A crotalária também exibiu elevada sensibilidade a presença do herbicida, constatando-se para dose $0,24 \mathrm{~kg} \mathrm{ha}^{-1}, 88 \%$ de fitotoxicidade aos 14 DAE, e 97\% aos 21 DAE (Figura 1). Pires, Procópio, Santos, Souza e Dias (2008) em estudo de fitorremediação de solo contaminado com tebuthiuron, utilizou C. juncea como planta indicadora em função da elevada suscetibilidade da espécie ao produto.

A braquiária e o sorgo (Figura 1), aos $7 \mathrm{DAE}$ não apresentaram sintomas visuais de fitotoxicidade maiores que $30 \%$ até a dose de $0,72 \mathrm{~kg} \mathrm{ha}^{-1}$. Em contrapartida, aos 21 DAE o incremento das doses causou o 


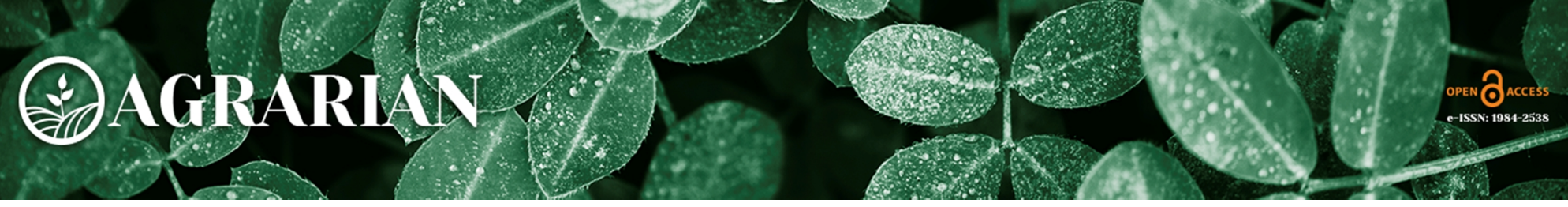

aumento da fitotoxicidade, constatando-se valores superiores a $90 \%$ a partir da dose de $0,72 \mathrm{~kg} \mathrm{ha}^{-1} \mathrm{e} 0,48 \mathrm{~kg}$ $\mathrm{ha}^{-1}$, respectivamente. Negrisoli, Velini, Rossi, Correia e Costa (2007) observaram que doses do herbicida tebuthiuron acima de $0,48 \mathrm{~kg} \mathrm{ha}^{-1}$ i.a., foram satisfatórias no controle de $U$. decumbens aos 21 dias após aplicação do herbicida em sistema de cana crua.

O amendoim foi a espécie que apresentou maior tolerância à presença de doses crescentes do herbicida no solo, sendo a única a não atingir o nível de $100 \%$ de fitotoxicidade mesmo na maior dose recomendada.

Tomate e crotalária foram às espécies analisadas que obtiveram os menores valores de MSPA, acumulando valor máximo de $32 \%$ na dose $0,12 \mathrm{~kg} \mathrm{ha}^{-1}$ (Figura 2), não sendo possível, portanto, obter a $\mathrm{GR}_{50}$. Pires et al. (2008) constataram que para doses elevadas de tebuthiuron (superiores à 1,0 $\mathrm{kg} \mathrm{ha}^{-1}$ ), não houve acúmulo de massa seca por $C$. juncea.

Amendoim (Arachis hypogaea)

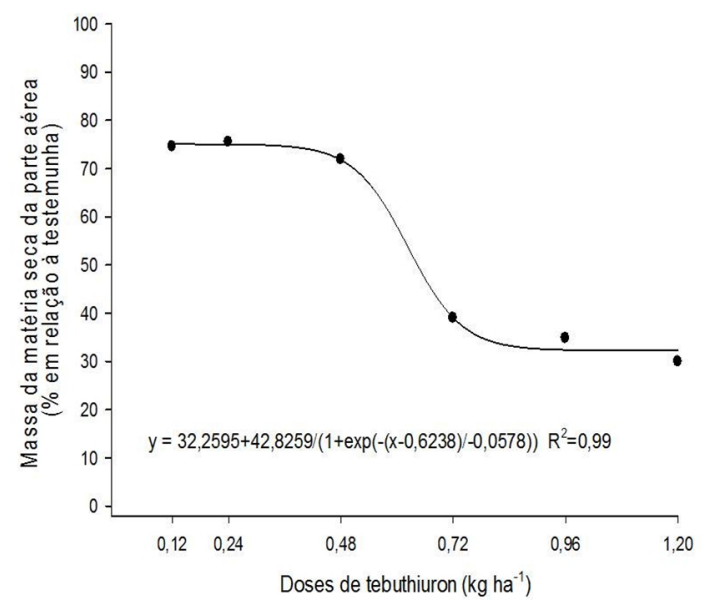

Braquiária (Urochloa brizantha)

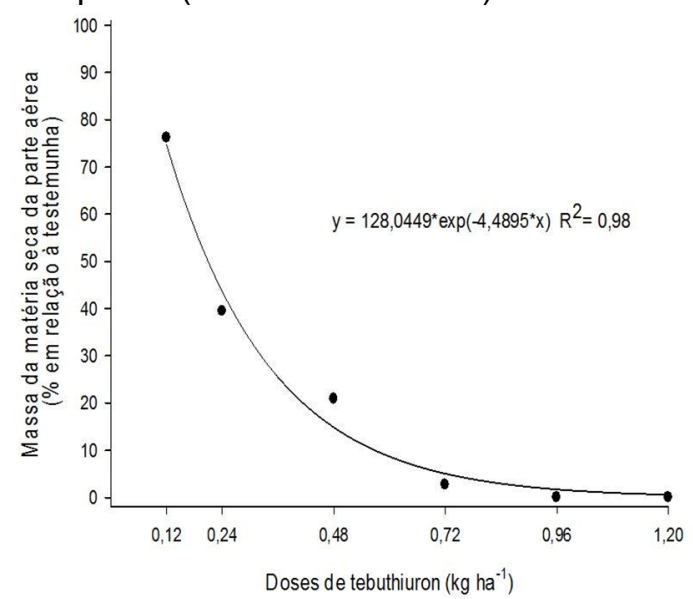

Pepino (Cucumis sativus)

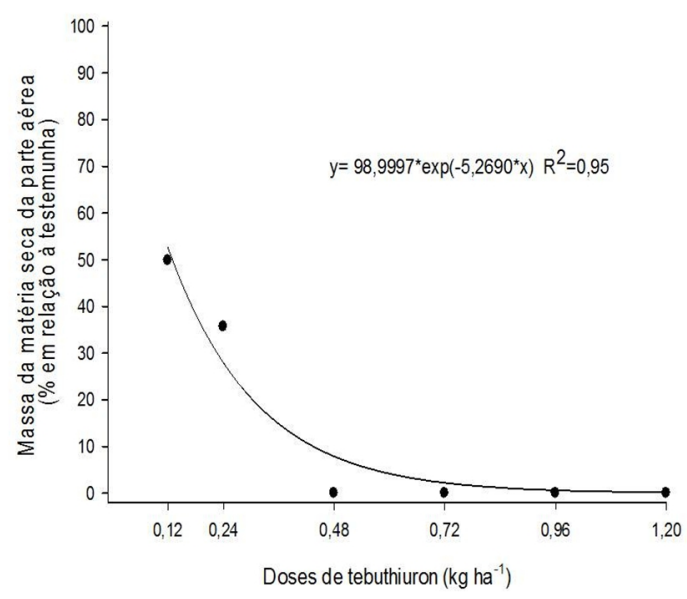

Aveia (Avena sativa)

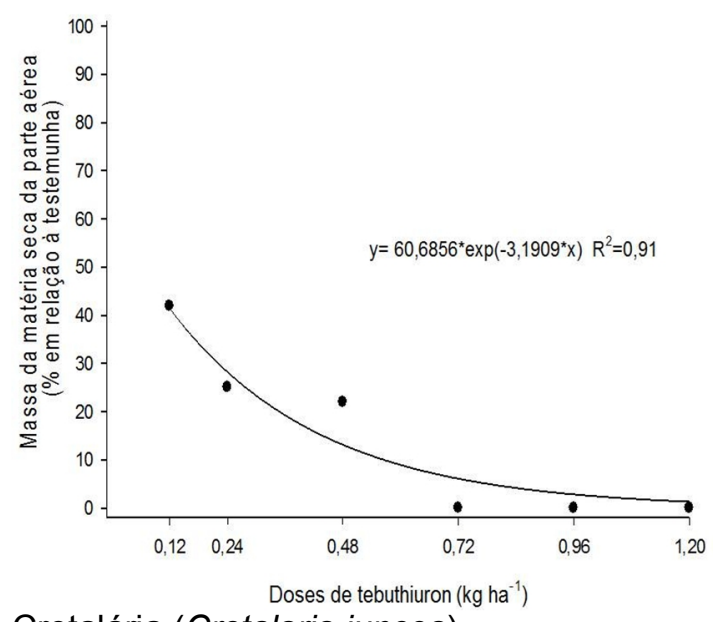

Crotalária (Crotalaria juncea)

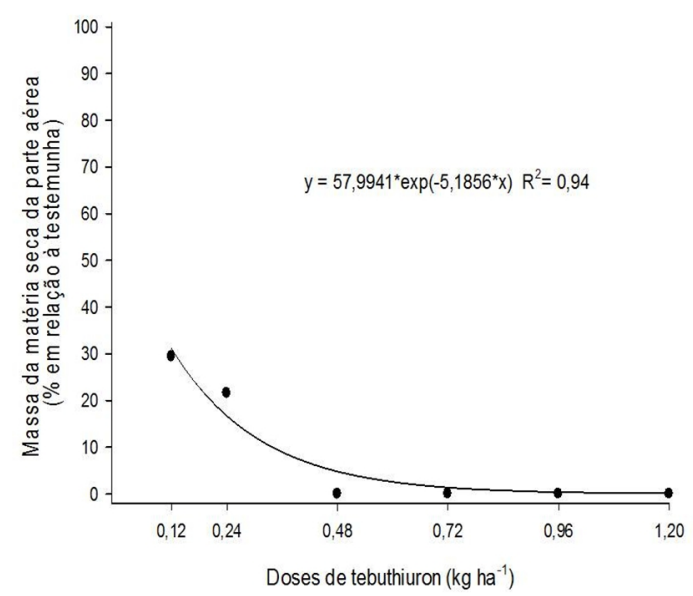

Sorgo (Sorghum bicolor)

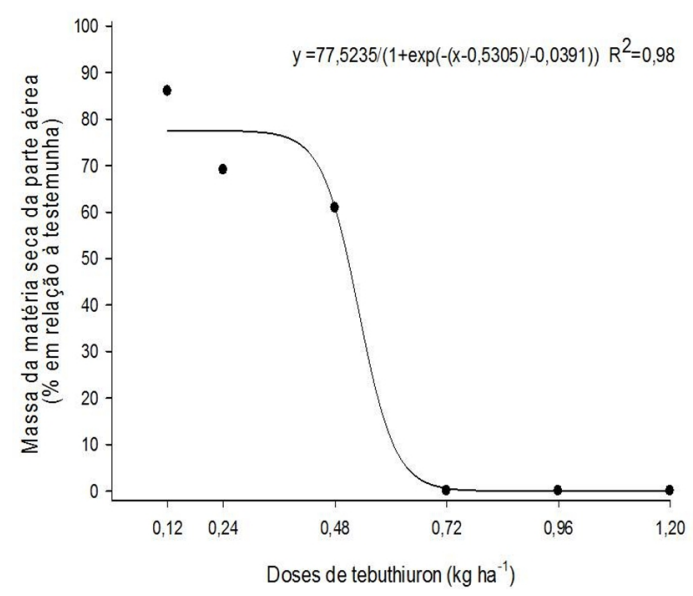


Sin

Tomate (Lycopersicon esculentum)

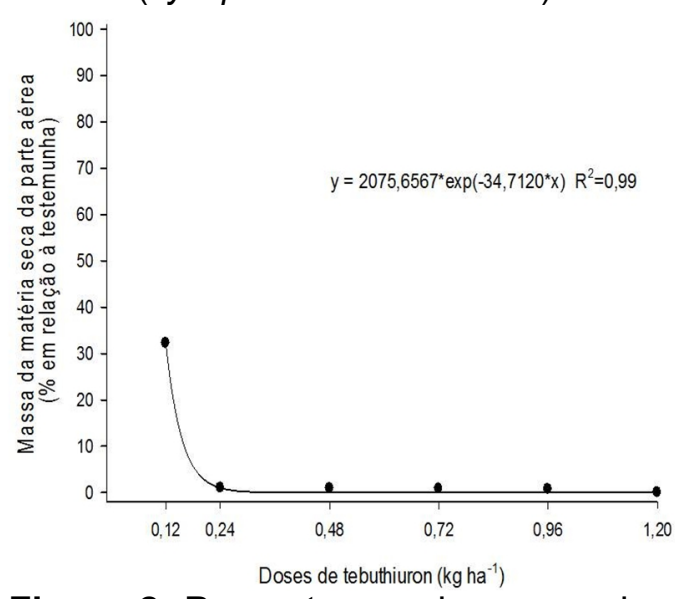

Figura 2. Porcentagem de massa da matéria seca da parte aérea (MSPA) em plantas de amendoim, aveia, braquiária, crotalária, pepino, sorgo e tomate com a aplicação de 10;20;40;60; 80 e 100\% da dose recomendada de tebuthiuron $\left(1,2 \mathrm{~kg} \mathrm{ha}^{-1}\right)$.

Notou-se diminuição exponencial na MSPA para o pepino e aveia, observando-se, as doses de 0,48 e $0,72 \mathrm{~kg} \mathrm{ha}^{-1}$ respectivamente, a redução foi de 8 e $2 \%$ de MSPA em relação à testemunha para o pepino e 13 e 6\% para a aveia (Figura 2). Este comportamento evidencia a sensibilidade das plantas em decorrência do incremento das doses do herbicida. Quanto à $\mathrm{GR}_{50}$, o pepino exibiu redução de $50 \%$ do acúmulo de massa com a dose de $0,13 \mathrm{~kg} \mathrm{ha}^{-1}$.

Para sorgo e braquiária o incremento das doses a partir de $0,72 \mathrm{~kg}^{-1}$ hão proporcionou acúmulo de MSPA, resultando em valores próximos de zero (Figura 2). Por meio das curvas sigmoidal e exponencial decrescente, verificou-se que a $\mathrm{GR}_{50}$ dessas espécies foi de 0,51 e $0,21 \mathrm{~kg} \mathrm{ha}^{-1}$, respectivamente. Brighenti et al. (2012) constataram valores menores de massa verde e seca de S. bicolor na presença de atrazine, molécula com mesmo mecanismo de ação do produto usado no presente trabalho, na dose $3,0 \mathrm{~kg} \mathrm{ha}^{-1}$ i.a.

A MSPA do amendoim apresentou comportamento sigmoidal, sendo a espécie menos sensível ao herbicida, não obtendo valores inferiores a 30\% de MSPA, mesmo nas doses mais elevadas do herbicida $\left(0,72,0,96\right.$ e $\left.1,2 \mathrm{~kg} \mathrm{ha}^{-1}\right)$. Para essa espécie a dose de $0,58 \mathrm{~kg} \mathrm{ha}^{-1}$ do herbicida provocou redução de $50 \%$ do acúmulo de massa (Figura 2). Tal tolerância diferencial pode possibilitar o uso da espécie como bioindicadora em estudos e monitoramento da presença do tebuthiuron no solo. Ademais, tendo em vista a produção de grãos e a função de adubo verde desempenhada pelo amendoim em sistemas de sucessão com a cana-deaçúcar (Lima, 2006), a variação no acúmulo de matéria seca observada nas plantas cultivadas em solo com a presença do herbicida tebuthiuron deve ser levada em consideração, assim como os impactos sobre a produtividade de grãos.

Sendo assim, com este trabalho foi possível a identificação de outras espécies, além do pepino, com potencial de uso como bioindicadoras da presença do herbicida tebuthiuron, haja vista o comportamento desta molécula no ambiente e, dessa forma, permitir o estudo e monitoramento do comportamento do tebuthiuron no solo.

\section{Conclusão}

Possuem potencial de uso como bioindicadora do tebuthiuron em ordem crescente de sensibilidade: amendoim, aveia, braquiária, sorgo e pepino.

\section{Referências}

Barizon, R.R.M., Lavorenti, A., Reginato, J.B., Prata, F., \& Tornisielo, V.L. (2006). Simulação do transporte e da sorção de imazaquin em colunas de solo. Revista Brasileira de Ciência do Solo, 30(4), 615-623. https://doi.org/10.1590/S0100-06832006000400002.

Blanco, F.M.G., \& Velini, E.D. (2005). Persistência do herbicida sulfentrazone em solo cultivado com soja e seu efeito em culturas sucedâneas. Planta Daninha, 23(4), 693-700. https://doi.org/10.1590/S010083582005000400018. 
Blanco, H.G., \& Oliveira, D.A. (1987). Persistência de herbicidas em Latossolo Vermelho-Amarelo em cultura de cana-de-açúcar. Pesquisa Agropecuária Brasileira, 22, 681-687.

Brighenti, A.M., Nicodemos, L.C., Calsavara, L.H.F., Martins, C.E., Rocha, W.S.D., \& Souza Sobrinho, F. (2012). Seletividade de Herbicidas à Cultura do Sorgo. In XXIX Congresso Nacional de Milho e Sorgo (p. 1092), Águas de Lindóia, SP.

Cadersa, Y., \& Gungadurdoss, M. (2010). Carryover of soil-applied herbicides on flue-cured tobacco. University of Mauritius Research Journal, 16.

Diesel, F., Trezzi, M.M., Pazuch, D., Xavier, E., Rosin, D., \& Pagnoncelli, F. (2012). Seleção de espécies da família Cucurbitaceae e Chenopodiaceae para indicação da presença de saflufenacil no solo. Pesticidas: Revista de Ecotoxicologia e Meio Ambiente, 22, 35-42. http://dx.doi.org/10.5380/pes.v22i1.30794.

Santos, H.G., Jacomine, P.K.T., Anjos, L.H.C., Oliveira, V.A., Lumbreras, J.F., Coelho, M.R., Almeida, J.A., Araujo Filho, J.C., Oliveira, J.B., \& Cunha, T.J.F. (2018). Sistema Brasileiro de Classificação de Solos. Brasília: Embrapa Solos.

Faria, A.T., Souza, M.F., Passos, A.B.R.J., Silva, A.A., Silva, D.V., Zanuncio, J.C., \& Rocha, P.R.R. (2018). Tebuthiuron leaching in three Brazilian soils as affected by soil pH. Environmental Earth Sciences, 77, 214.

Faria, A.T., Saraiva, D.T., Pereira, A.M., Rocha, P.R.R., Silva, A.A., Silva, D.V., Ferreira, E.A., \& Silva, G.S. (2014). Efeitos de herbicidas na atividade da microbiota rizosférica e no crescimento da cana-de-açúcar. Bioscience Journal, 30(4), 1024-1032.

Faria, A.T. (2013). Sorção, dessorção, meia-vida e lixiviação do tebuthiuron em latossolos brasileiros (Dissertação de Mestrado, Universidade Federal de Viçosa, Viçosa).

Fernandes, C.P.C., Braz, A.J.B.P., Procopio, S.O., Dan, H.A., Braz, G.B.P., Barroso, A.L.L., Menezes, C.C. E., Simon, G.A., \& Braz, L.B.P. (2011). Tolerância do feijoeiro a herbicidas aplicados na cultura da cana-deaçúcar. Revista Brasileira de Herbicidas, 10(2), 121-133.

Freitas, M.A.M., Passos, A.B.R.J., Torres, L.G., Moraes, H.M.F., Faustino, L.A., Rocha, P.R.R., \& Silva, A.A. (2014). Sorção do sulfentrazone em diferentes tipos de solo determinada por bioensaios. Planta Daninha, 32(2), 385-392. https://doi.org/10.1590/S0100-835820140002000.

Gazziero, D.L.P., Karan, D.,Voll, E., \& Ulbrich, A. (1997). Persistência dos herbicidas imazaquin e imazethapyr no solo e os efeitos sobre plantas de milho e pepino. Planta Daninha, 15(2). https://doi.org/10.1590/S0100-83581997000200009.

Gomes, M.A.F., Spadotto, C.A., Pereira, A.S., Matallo, M.B., \& Luchini, L.C. (2006). Movimento do herbicida tebuthiuron em dois solos representativos das áreas de recarga do aquífero Guarani. Revista Brasileira de Engenharia Agrícola e Ambiental, 10(2), 479-483. https://doi.org/10.1590/S1415-43662006000200032.

Gomes, M.A.F.,Spadotto, C.S., \& Lanchotte, V.L. (2001). Ocorrência do herbicida tebuthiuron na água subterrânea da microbacia do córrego espraiado, Ribeirão Preto - SP. Revista Ecotoxicologia e Meio Ambiente, 11, 65-76.

Guerra, N. Oliveira Júnior, R.S., Constantin, J., Oliveira Neto, A.M., Gemelli, T.M.C., \& Guerra, A. (2016). Potencial de lixiviação de herbicidas utilizados na cultura da canade-açúcar. Revista Campo Digital, 11(1), 4253.

Guerra, N., Oliveira Júnior, R.S., Constantin, J., Oliveira Neto, A.M., Dan, H.A., Alonso, D.G., \& Jumes, T.M.C. (2011). Seleção de espécies bioindicadoras para os herbicidas trifloxysulfuron-sodium e pyrithiobac-sodium. Revista Brasileira de Herbicidas, 10(1), 37-48. https://doi.org/10.7824/rbh.v10i1.89.

Hijano, N. (2016). Interferência de capim-camalote em cana-de-açúcar e seletividade de indaziflam e indaziflam + metribuzin aplicados em cana-de-açúcar no sistema MPB (Tese de Doutorado, Universidade Estadual Paulista, Jaboticabal).

Hutchinson, P.J., Morishita, D.W., \& Price, W.J. (2007). Season-long dose-response of potato to sulfometuron. Weed Science, 55(5), 521- 527. 
Inoue, M.H., Santana, C.T.C., Oliveira Júnior., R.S., Possamai, A.C.S., Santana, D.C., Arruda, R.A.D., Dallacort, R., \& Sztoltz, C.L. (2011). Efeito residual de herbicidas aplicados em pré-emergência em diferentes solos. Planta Daninha, 29(2), 429-435. https://doi.org/10.1590/S0100-83582011000200021.

Lima, E.A. (2006). Avaliação fitotécnica e econômica de cultivares de soja em área de reforma de cana-deaçúcar, na região Norte Fluminense (Tese de Doutorado, Universidade Estadual do Norte Fluminense Darcy Ribeiro, Campos dos Goytacazes).

Madalão, J.C., Pires, F.R., Chagas, K., Cargnelutti Filho, A., \& Procópio, S.O. (2012). Uso de leguminosas na fitorremediação de solo contaminado com sulfentrazone. Pesquisa Agropecuária Tropical, 42(4), 390-396. https://doi.org/10.1590/S1983-40632012000400001.

Marchesan, E.D., Dedordi, G., Rezzi, M.M., Vidal, R.A., \& Dick, D.P. (2011). Seleção de espécies bioindicadoras para uso em bioensaios de lixiviação e persistência de atrazina no solo. (2011). Pesticidas: Revista de Ecotoxicologia e Meio Ambiente, 21, 47-54. http://dx.doi.org/10.5380/pes.v21i0.25937.

Melo, C.A.D., Medeiros, W.N., Tuffi, L.D.S., Ferreira, F.A., Tiburcio, R.A.S., \& Ferreira, L.R. (2010). Lixiviação de sulfentrazone, isoxaflutole e oxyfluorfen no perfil de três solos. Planta Daninha, 28(2), 385-392. https://doi.org/10.1590/S0100-83582010000200018.

Mendes, K.F., Souza, T.N.R., Possamai, A.C.S., Inoue, M.H., Nunes, A.K.A., \& Mertens, T.B. (2015). Seleção de plantas indicadoras para o monitoramento do mesotrione e metribuzin em solo argiloso. Revista de Ciências Agroambientais, 13(1), 53- 59.

Mendes, K.F., Inoue, M.H., Matos, A.K.A., Possamai, A.C.S., Tschope, M.C., Goulart, B.F., \& Ben, R. (2012). Seleção de bioindicadores para monitoramento da mobilidade e persistência de herbicidas aplicados no solo. Revista Brasileira de Herbicidas, 11(2), 213-221. https://doi.org/10.7824/rbh.v11i2.156.

Negrisoli, E., Velini, E.D., Rossi, C.V.S.; Correia, T.M., \& Costa, A.G.F. (2007). Associação do herbicida tebuthiuron com a cobertura de palha no controle de plantas daninhas no sistema de cana-crua. Planta daninha, 25(3), 621-628. https://doi.org/10.1590/S0100-83582007000300023.

Nunes, A.L., \& Vidal, R.A. (2009). Seleção de plantas quantificadoras de herbicidas residuais. Pesticidas: Revista de Ecotoxicologia e Meio Ambiente, 19(1), 19-28. http://dx.doi.org/10.5380/pes.v19i0.16550.

Peñaherrera-Colina, L.A., Souza, I.F., Guilherme, L.R.G., \& Bueno Filho, J.S.S. (2005). Persistência biológica de ametryn, diuron e oxyfluorfen no solo. Ciência agrotecnologia, 29(5), 980-987. https://doi.org/10.1590/S1413-70542005000500010.

Perim, L., Toledo, R.E.B., Negrisoli, E., Corrêa, M.R., Carbonari, C.A. Rossi, C.V.S., \& Velini, E.D. (2009). Eficácia do herbicida amicarbazone no controle em pós-emergência de espécies de corda-de-viola (Ipomoea grandifolia e Merremia cissoids). Revista Brasileira de Herbicidas, 8(1), 19-26. https://doi.org/10.7824/rbh.v8i1.64.

Pires, F.R., Souza, C.M., Silva, A.A., Queiroz, M.E. L.R., Procópio, S.O., Santos, J.B., Santos, E.A., \& Cecon, P.R. (2003). Seleção de plantas com potencial para fitorremediação de tebuthiuron. Planta Daninha, 21(3), 451-458. https://doi.org/10.1590/S0100-83582003000300014.

Pires, F.R., Procópio, S.O., Santos, J.B., Souza, C.M., \& Dias, R.R. (2008). Avaliação da fitorremediação de tebuthiuron utilizando Crotalaria juncea como planta indicadora. Revista Ciência Agronômica, 39(02), 245250.

Silva Junior, A.C., Gonçalves, C.G., Queiroz, J.R.G.Q., \& Martins, D. (2018). Evaluation of leaching potential of tebuthiuron using bioindicator plants. Arquivos do Instituto Biológico, 85, 1-9. https://doi.org/10.1590/18081657000692015.

SigmaPlot 12.0. (2011). Scientific Data Analysis and Graphing Software. http://www.sigmaplot.co.uk/products/sigmaplot/produpdates/prod-updates5.php.

Velini, E.D., Osipe, R., \& Gazziero, D.L.P. (2002). Procedimentos para instalação, avaliação e análise de experimentos com herbicidas. Sociedade Brasileira da Ciência das Plantas Daninhas (SBCPD), Londrina, PR.

Vidal, A., \& Vidal, R.A. (2009). Seleção de plantas quantificadoras de herbicidas residuais. Pesticidas: Revista de Ecotoxicologia e Meio Ambiente, 19, 19-28. http://dx.doi.org/10.5380/pes.v19i0.16550. 


\section{Pagrarial}

Teixeira, P.C., Donagemma, G.K., Fontana, A., \& Teixeira, W.G. (2017). Manual de Métodos de Análise de Solo. Brasília: Embrapa Solos.

The Pesticide Properties Data Base (PPDB). (2019). Pesticide, BioPesticideandVeterinarySubstancespropertiesdatabases. https://sitem.herts.ac.uk/aeru/ppdb/.

Walorczyk, S., Drożdżyński, D.; Kowalska, J.; Remlein-Starosta, D.; Ziółkowski, A.; Przewoźniak, M.; Gnusowski, B. (2013). Pesticide residues determination in Polish organic crops in 2007-2010 applying gas chromatography-tandem quadrupole mass spectrometry. Food Chemistry, 139(1), $482-487$. 10.1016/j.foodchem.2013.01.013. 